archives

of thermodynamics

Vol. 34(2013), No. 3, 161-172

DOI: 10.2478 /aoter-2013-0022

\title{
The thermal-flow behavior of the working chamber in an oil-free scroll compressor
}

\section{JÓZEF RAK*}

Wroclaw University of Technology, Institute of Heat Engineering and Fluid Mechanics, Wybrzeże Wyspiańskiego 27, 50-370 Wrocław, Poland

\begin{abstract}
The paper presents the full transient, two-dimensional finite volume method numerical calculations of the classical involute scroll compressor geometry. The purpose of the study was to develop and evaluate an adaptable implementation of numerical fluid mechanics and thermodynamics modeling procedure with a mesh deformation. The methodology consisting in the compression chamber geometry preparation, mesh generation and governing equations solving was described. The evaluation was carried by simulating an adiabatic compression process and the results were compared with the theoretical zero-dimensional model and the existing research concerning the scroll chamber computational fluid dynamics modeling. It has been shown that the proposed modeling routine results in good accuracy for the scroll compressors study applications.
\end{abstract}

Keywords: CFD; Scroll; Mesh deformation

\section{Nomenclature}

e $\quad-$ eccentricity

$g \quad-\quad$ spiral thickness

$r \quad-\quad$ radius of the evolute

$x, y \quad-\quad$ Cartesian coordinate

\section{Greek symbols}
$\alpha-$ involute angle
$\xi \quad-\quad$ scroll chamber parameter

\footnotetext{
*E-mail: jozef.rak@pwr.wroc.pl
} 
$\eta \quad-\quad$ scroll chamber parameter

$\Theta \quad-$ shaft rotation angle

\section{Introduction}

Scroll compressors belong to the family of rotary gas power machines [1]. The working principle of the scroll compressors is presented in Fig. 1. It consists in the cooperation of two spiral elements. The eccentric movement of one of them causes the cyclic compression chamber forming and moving toward the center of the device where the discharge is placed. The second element remains stationary.

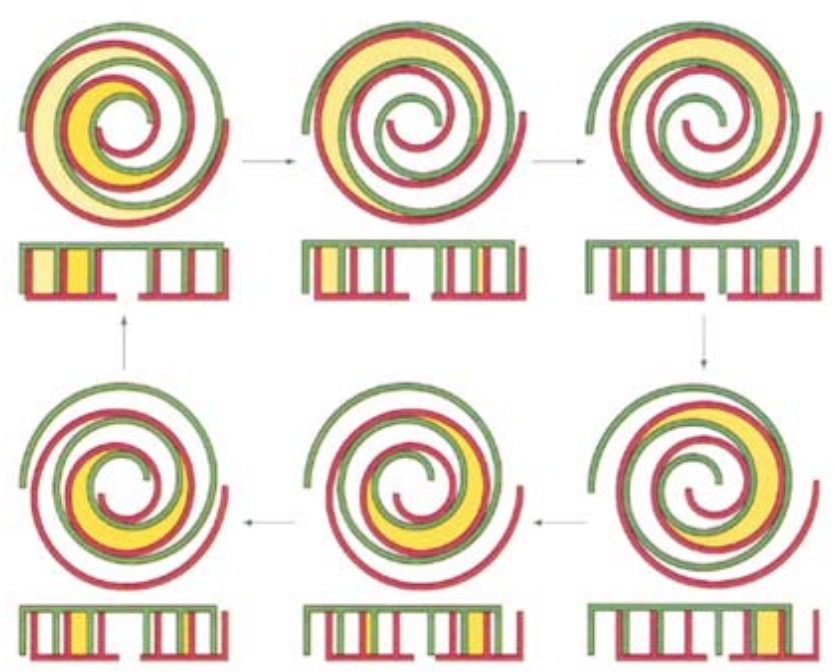

Figure 1. The scroll compressor working principle [2].

The blood circulation assistance concept is based on the use of pneumatic impulses to power the artificial heart ventricles. Scroll compressor design has many features that qualify it for the use in an external power supplied artificial heart. First of all such devices have a small number of moving parts compared to conventional compressors. This makes it possible to achieve a high reliability and longevity. This feature is important because of the need for long-term and permanent life support. Due to the proposed application the scroll compressors have other advantages. In the device there are a couple of working chamber pairs separated from each other. Therefore, the forces acting on the system are distributed in a uni- 
form manner. Additional force induced by eccentricity is balanced by the counterweights located on the shaft. The design is also free of suction and discharge valves. The effects of these design features are as follows: the small size of the unit, low vibration and noise, and the monotony of work. This means patient comfort, allows them to maintain mobile. It is therefore possible to put the whole unit in a suitcase or backpack that allows movement and performing many daily living activities.

In the field of scroll machines there were many approaches to describe the efficiency, thermodynamic state of medium as a function of an angle of the shaft rotation, e.g., [3-5]. These were very helpful in estimating and improving the scroll machinery although they were limited in terms of knowledge about medium behavior. More detailed information about the nature of the process (e.g., leakage, heat transfer, dynamic properties) may be obtained by using numerical methods. Thus due to the scroll machines nature the calculations have to be either quasi-transient or fully transient. Several papers were presented in this field. Pietrowicz et al. [6] or Ooi and Zhu [7] studies consisted is the analyses of a significant number of models of fluid dynamics and thermodynamics in the working chamber. Recently Pereira [8] presented new models of heat transfer and two chambers simulation.

The paper presents the full transient, two-dimensional finite volume method numerical calculations of the classical involute scroll compressor geometry. The purpose is to develop and evaluate an adaptable implementation of numerical fluid mechanics and thermodynamics modeling procedure with a mesh deformation. It can be very much useful during the studies on the shape and design optimization before the prototype stage. From computational point of view the approach is similar to the positive displacement machinery modeling [9]. The main objective in these kind of research is to create and properly deform the numerical grid and ensure that the conservation laws are satisfied during the time stepping [10]. Having satisfied the benchmark results it is possible to use this new scroll chamber modeling procedure in more complex cases with sophisticated boundary conditions and designs. Calculations consists the following phases: making the geometry and a numerical mesh, defining the boundary and initial conditions, solving the differential equations and the postprocessing. 


\subsection{Geometry}

The vane shape is described by Eq. (1) and it is shown in Fig. 2.

$$
\left\{\begin{array}{l}
x(\alpha)=r(\cos \alpha+\alpha \sin \alpha) \pm \frac{g}{2} \sin \alpha, \\
y(\alpha)=r(\sin \alpha+\alpha \cos \alpha) \pm \frac{g}{2} \sin \alpha .
\end{array}\right.
$$

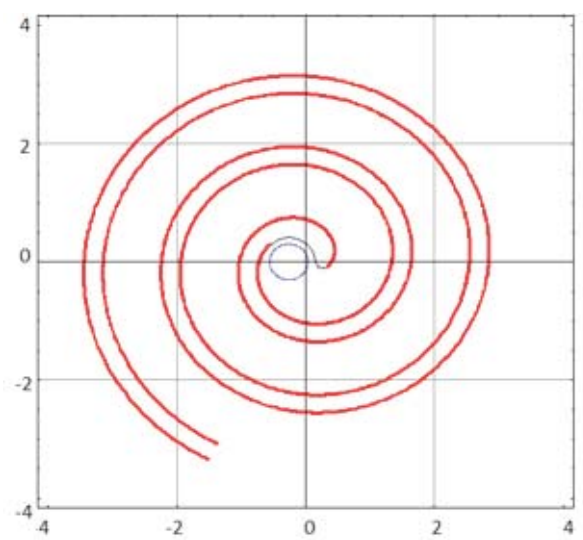

Figure 2. Spiral geometry.

Radius, $r$, of the evolute is $1.90 \mathrm{~mm}$. Spiral thickness (the length between involutes) $g$ is $3 \mathrm{~mm}$. In the suction area the vane is ended by two arcs and in the discharge area the involutes are linked by a straight line. The orbiting scroll is rotated $180^{\circ}$ about the fixed one and shifted by the eccentricity, $e$. There is no clearance between vanes so that value is $e=r \pi$. The pair of scrolls designed in that way makes a machine with a number of common points, between which there are working chambers.

A single working chamber of the scroll machine, which is based on the classical geometry, can be described by using the parametrical equations [3]

$$
\begin{gathered}
x(\xi, \eta, \Theta)=r(\sin \xi \xi \cos \xi)+e(\sin \Theta+\eta(\cos \xi-\sin \Theta)), \\
y(\xi, \eta, \Theta)=-r(\cos \xi \xi \sin \xi)+e(\sin \Theta+\eta(\sin \xi-\cos \Theta)),
\end{gathered}
$$

where:

$\xi \quad$ - parameter in the range from $\frac{\pi}{2}-\Theta-2 \pi(n-1)$ to $2.5 \pi-\Theta-2 \pi(n-1)$,

$\eta \quad$ - parametr defining the boundary, for orbiting $(\eta=1)$ or fixed $(\eta=1)$ scroll,

$\Theta \quad-$ angle of rotation. 
Above equations were used to generate the geometry of the chamber.

\subsection{The mesh}

Planning and creating a numerical grid suitable for the task is a key issue in the calculations. A hexagonal grid has been chosen. It was motivated by a very complex geometry of the domain and by its properties in terms of the mesh deformation required. In the $z$ direction the mesh is one element thick. The numerical grid (Fig. 3) was made based on the geometry, and defined areas of increased density. During the simulation, if the mesh quality has become too low, the remeshing script was ran and the results were interpolated into a new one that was matching the actual geometry of the chamber. In order to preserve the mesh topology (number of nodes cells, faces and their arrangement) and ensure that the new generated grid is always consistent with the previous one, the automatic script was made. This procedure ensures that the transient time-stepping, mesh deformation and remising is about to develop numerically stable solution.

The mesh preparations were made using the ANSYS ICEM 14.5 software [11] with its blocking functionality. Using the geometry data, the script was generating the structured mesh containing 5632 elements with a prescribed topology.

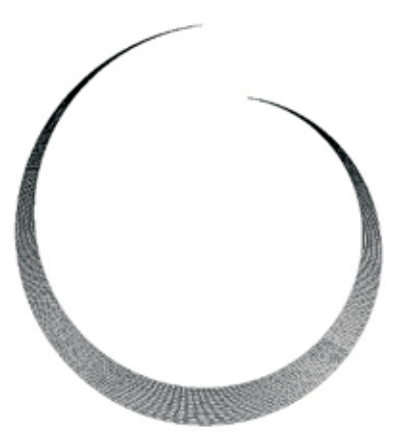

a)

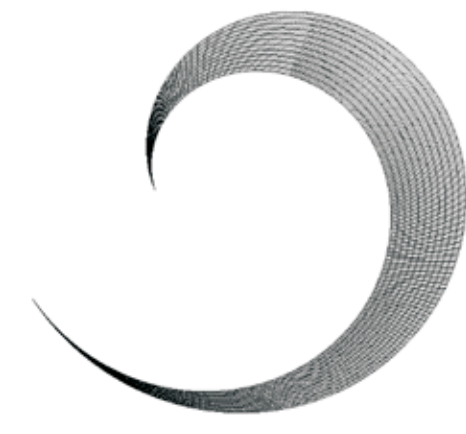

b)

Figure 3. Spiral geometry: a) $\Theta=0.07 \pi$, b) $\Theta=1.68 \pi$.

\subsection{Boundary and initial conditions}

In order perform the calculations the following boundary and initial conditions were made: 
- the working fluid is air treated as an ideal gas,

- thermodynamically the boundaries are adiabatic,

- fluid velocity at the fixed wall is zero and orbiting scroll wall has a no-slip condition,

- angular velocity of the shaft is $3000 \mathrm{rpm}$.

Turbulent flow calculations were performed by using the SST $k-\varepsilon$ turbulence model [11]. The choice was made due to the nature of the geometry where there are two different kinds of areas. In the central part of the chamber the distance between the walls is high, while the surroundings of common points are long and narrow. The simulation was made for such rotary angle range to make sure that the chamber working regime is between suction and discharge where the volume change is linear [3]. The area of interest is shown in Fig. 4 as a compression volume.

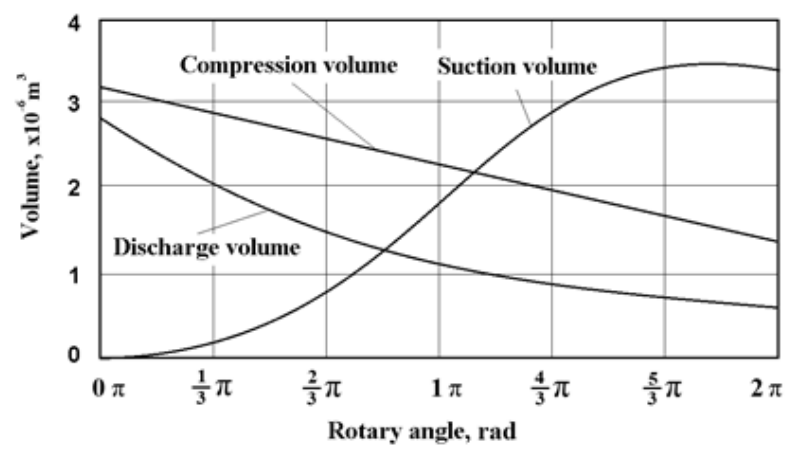

Figure 4. Volume changes in the areas of scroll machine.

\section{Results and disscussion}

The finite volume analysis based on the described principles was carried out with the commercial ANSYS CFX 14.5 solver software [12] on the SuperNova computing cluster in Wroclaw Centre for Networking and Supercomputing at Wroclaw University of Technology. The results for the three rotation angles $(0.08 \pi, 1.68 \pi$, and $2.18 \pi)$ are presented. The total pressure, temperature and velocity are shown in Figs. 5, 6, and 7. In all of the cases there is a distinct nonuniform distribution of variables. The highest gradients can be found in the common points areas while in the central part 
the values are more homogeneous. The pressure field shows that during the whole process the values differentiates along the $\xi$ parameter and it becomes more uniformly distributed as the crank angle rises. The temperature distribution is more dependent on the wall distance and it is considerably higher in the central area than at the walls. The values are also convergent with the zero-dimensional adiabatic compression. The velocity field is as expected for the defined type of motion. The fluid is being pushed, by a 'squish motion' affected by the orbiting scroll, from the first to the second common point.

Since the complex mesh deformation in the difficult topology circumstances the simulation was made in fact with a Key-Frame remeshing method [9]. Interpolating the results into the new numerical grid carries an increased risk of generating an artificial mass source due to violation of space conservation equation $[9,10]$. In the calculations presented in this paper the mass conservation error is lower than $1 \%$. This may lead to the conclusion that mass generation was avoided.

Presented presssure, temperature and velocity fields results are comparable with earlier papers concerning discussed topic [6,7]. In those studies the fluid behaviour was similar in principle. Additionally in Fig. 8 there is a comparison between the average total pressure and the theoretical pressure of the adiabatic compression process. These zero-dimensional values show the expected trend in the function of rotation angle.

\section{Conclusions}

The aim of this paper was to develop and evaluate a platform to study the thermodynamic, flow and dynamics of spiral machines properties with the use of commercial software. A flexible procedure for verifying different solutions in this area was created. The evaluation and the comparison with the results from the literature shows that the approach is correct and may by successfully used in the further considerations. The proposed method allows to extend the calculations by a more complex cases that consists phenomenons such as axial and radial leakage, the full three-dimensional simulation taking into account the influence of buoyancy or other working chambers formed in the machine. It also allows testing other engineering solutions in the field of a scroll geometry, cooling systems, etc. on the conceptual developing phase. Results are promising and will allow the development in the area of modeling and optimization in the field of scroll 
a)

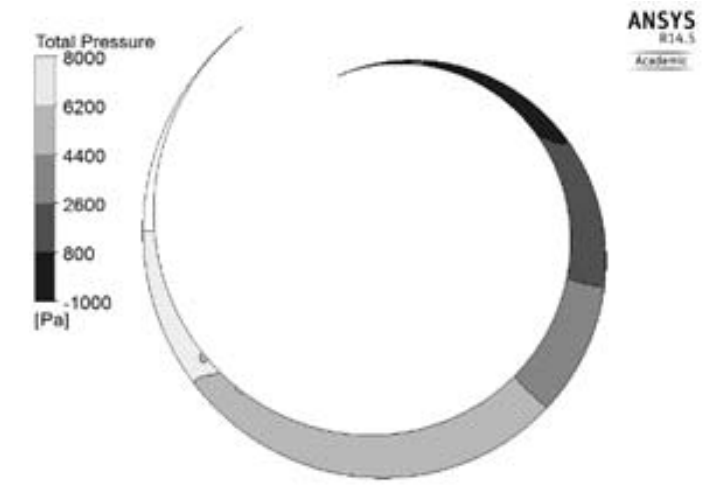

b)

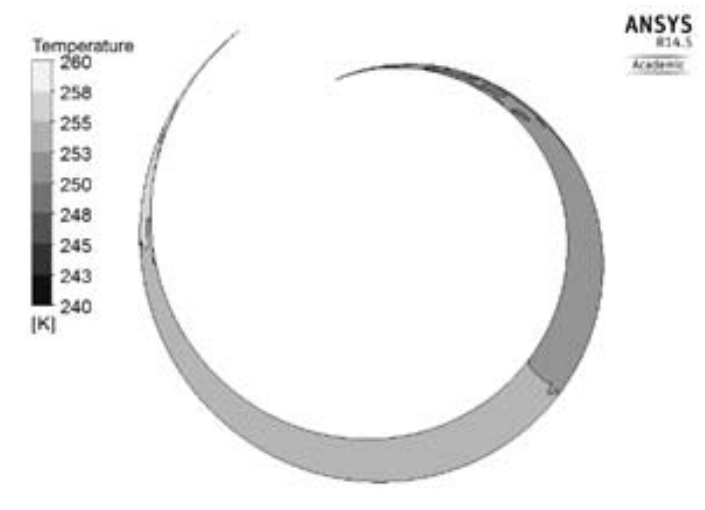

c)

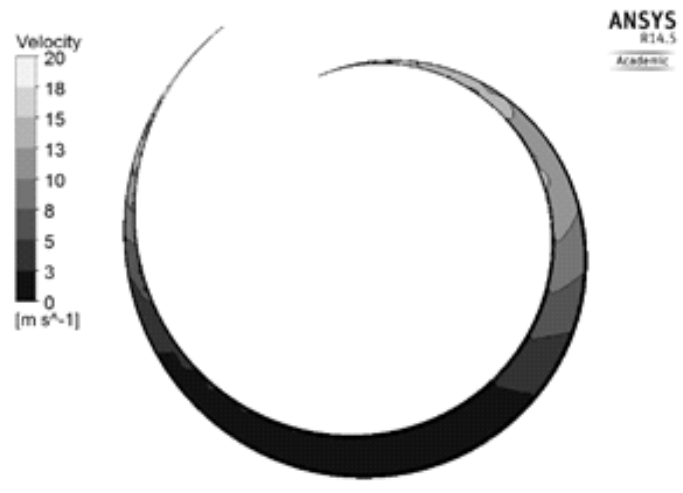

Figure 5. Spiral geometry: a) total pressure, b) temperature, c) velocity. 
a)

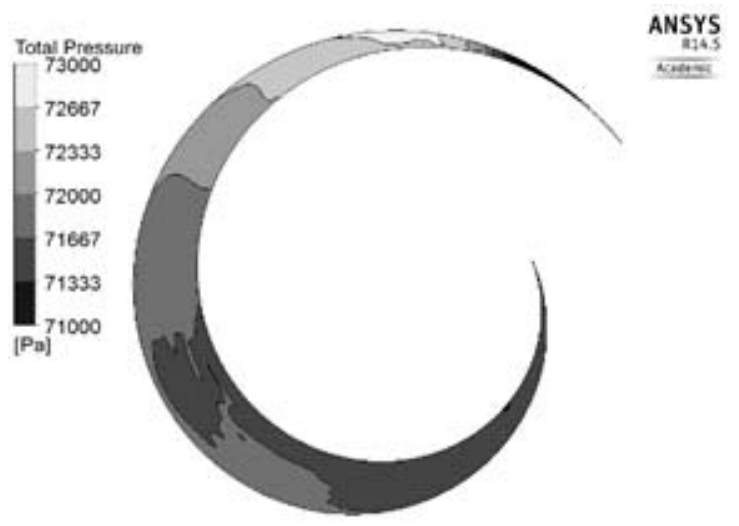

b)

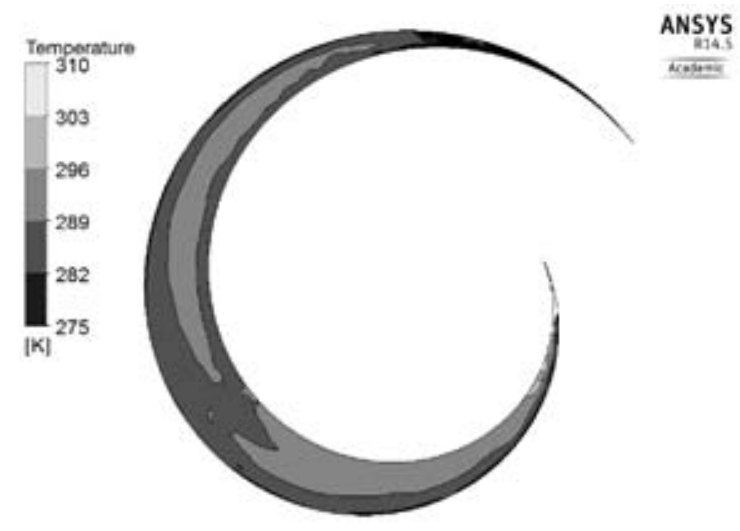

c)

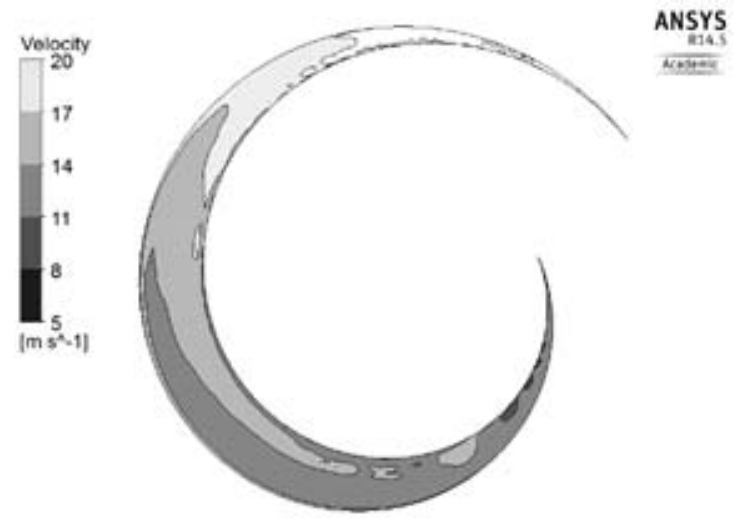

Figure 6. The distribution of pressure (a), temperature (b), and velocity (c) at the angle of $0.08 \pi$ 
a)

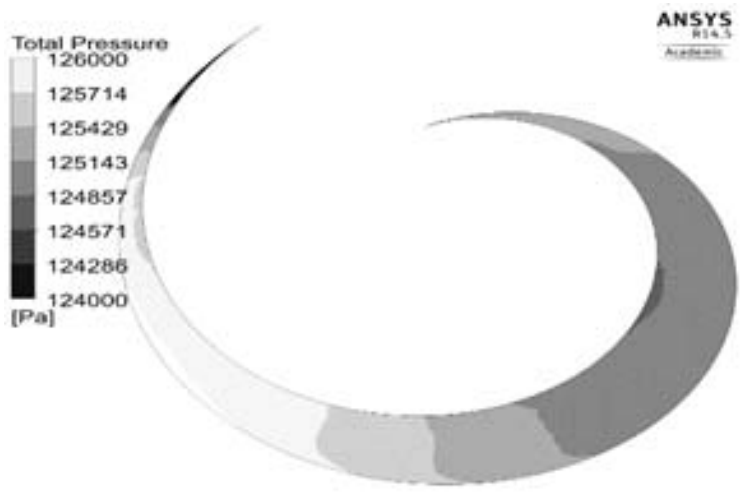

b)

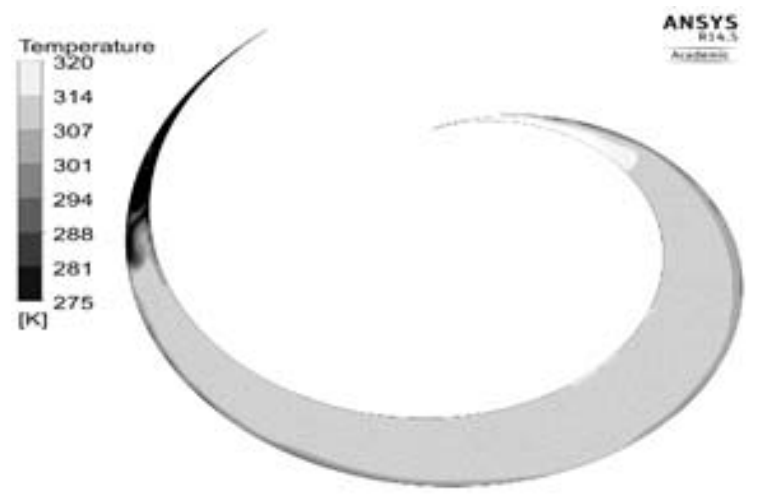

c)

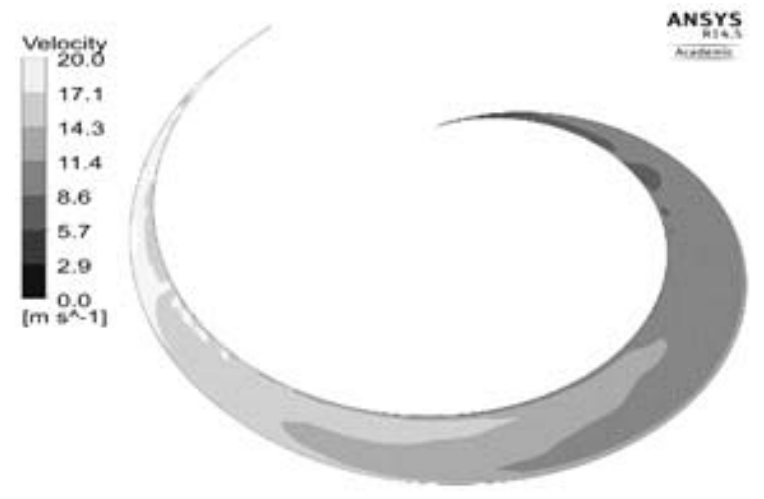

Figure 7. The distribution of pressure (a), temperature (b), and velocity (c) at the angle of $1.68 \pi$. 


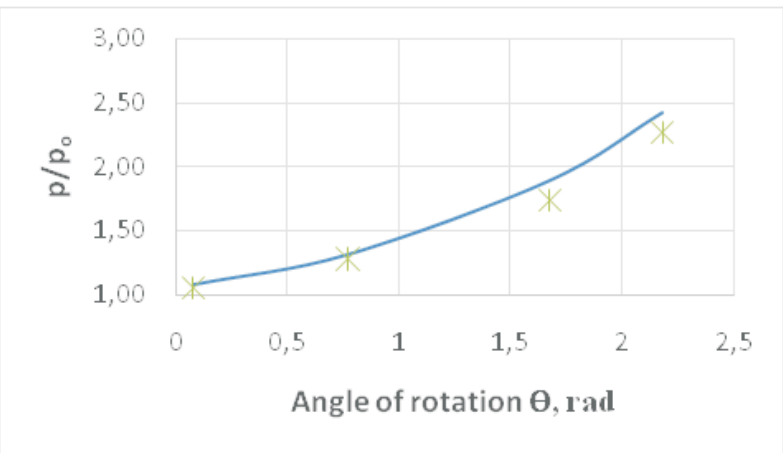

Figure 8. The comparison between the obtained results and theoretical adiabatic compression curve.

machines. It may be applied to develop the extracorporeal supply of the artificial heart project that requires a reliable, small and easy in maintenance compressing device [13]. The model studies may lead to progress in this field thus they should be continued.

Received 8 July 2013

\section{References}

[1] Gnutek Z.: Rotating Blade Machinery. Wrocław 1997 (in Polish).

[2] Gravesen J., Henriksen C.: The geometry of the scroll compressor. SIAM Rev. 43(2001), 1, 113-126.

[3] Chen Y., Halm N.P., Groll E.A., Braun J.E.: A comprehensive model of scroll compressors. Part I:: Compression process modeling. Int. Compressor Engineering Conf. (2000). Paper 1455.

[4] Morishita E., Sugihara M., Inaba T., Nakamura T.: Scroll compressor analytical model. Int. Compressor Engineering Conf. (1984). Paper 495.

[5] Blunier B. Cirrincione G., Miraoui A.: Novel geometrical model of scroll compressors for the analytical description of the chamber volumes. Int. Compressor Engineering Conf. (2006). Paper 1745.

[6] Pietrowicz S., Yanagisawa T., Funuta M., Gnutek Z.: Mathematical modeling of physical processes in the scroll compressor chamber. Int. Compressor Engineering Conf. (2002). Paper 1589.

[7] Oor K.T., Zhu J.: Convective heat transfer in a scroll compressor chamber: a 2-D simulation. Int. J. Therm. Sci. 43(2004), 677-688. 
[8] Pereira E., LAnge L.: Modeling and analysis of leakage and heat transfer in scroll compressors. Calor Em Compressores Scroll, Federal University of Santa Catarina, Ph.D. Thesis, Florianópolis, March 2012 (in Portuguese).

[9] Rane Sh., Kovacevic A., Kethidi M.: CFD modelling in screw compressors with complex multi rotor configurations. International Compressor Engineering Conference 2012.Paper 2141.

[10] Ferziger J.H., Peric M.: Computational Methods for Fluid Dynamics. Springer, Berlin 1996.

[11] Menter F.R.: Zonal two equation $k-\omega$ turbulence models for aerodynamic flows. AIAA Paper 93-2906(1993).

[12] ANSYS Academic Research, Release 14.5, Help System, ANSYS, Inc.

[13] Czak M.: Polish Program Artificial Heart. Bull. 4(2010). 